\title{
Portable double-sided pulsed laser heating system for time-resolved geoscience and materials science applications
}

G. Aprilis, C. Strohm, I. Kupenko, S. Linhardt, A. Laskin, D. M. Vasiukov, V. Cerantola, E. G. Koemets, C. McCammon, A. Kurnosov, A. I. Chumakov, R. Rüffer, N. Dubrovinskaia, and L. Dubrovinsky

Citation: Review of Scientific Instruments 88, 084501 (2017);

View online: https://doi.org/10.1063/1.4998985

View Table of Contents: http://aip.scitation.org/toc/rsi/88/8

Published by the American Institute of Physics

\section{Articles you may be interested in}

Novel diamond cells for neutron diffraction using multi-carat CVD anvils

Review of Scientific Instruments 88, 083905 (2017); 10.1063/1.4997265

High-pressure rotational deformation apparatus to $135 \mathrm{GPa}$

Review of Scientific Instruments 88, 044501 (2017); 10.1063/1.4979562

Combined laser ultrasonics, laser heating, and Raman scattering in diamond anvil cell system

Review of Scientific Instruments 87, 123908 (2016); 10.1063/1.4972588

New developments in laser-heated diamond anvil cell with in situ synchrotron x-ray diffraction at High Pressure Collaborative Access Team

Review of Scientific Instruments 86, 072201 (2015); 10.1063/1.4926895

Time differentiated nuclear resonance spectroscopy coupled with pulsed laser heating in diamond anvil cells Review of Scientific Instruments 86, 114501 (2015); 10.1063/1.4935304

The world's smallest capacitive dilatometer, for high-resolution thermal expansion and magnetostriction in high magnetic fields

Review of Scientific Instruments 88, 083903 (2017); 10.1063/1.4997073

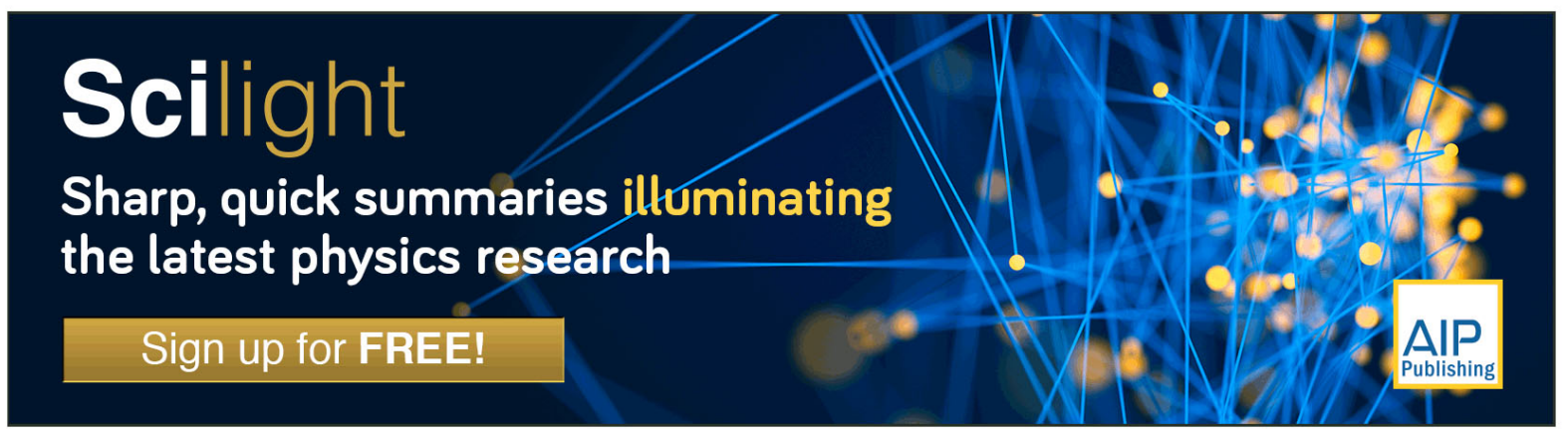




\title{
Portable double-sided pulsed laser heating system for time-resolved geoscience and materials science applications
}

\author{
G. Aprilis, ${ }^{1,2, a)}$ C. Strohm,${ }^{3}$ I. Kupenko, ${ }^{4,5}$ S. Linhardt, ${ }^{1}$ A. Laskin, ${ }^{6}$ D. M. Vasiukov,,${ }^{1,2}$ \\ V. Cerantola, ${ }^{1,5}$ E. G. Koemets, ${ }^{1}$ C. McCammon, ${ }^{1}$ A. Kurnosov, ${ }^{1}$ A. I. Chumakov, ${ }^{5}$ R. Rüffer, ${ }^{5}$ \\ N. Dubrovinskaia, ${ }^{2}$ and L. Dubrovinsky ${ }^{1}$ \\ ${ }^{1}$ Bayerisches Geoinstitut, Universität Bayreuth, D-95440 Bayreuth, Germany \\ ${ }^{2}$ Materials Physics and Technology at Extreme Conditions, Laboratory of Crystallography, Universität Bayreuth, \\ D-95440 Bayreuth, Germany \\ ${ }^{3}$ Photon Science, DESY, D-22607 Hamburg, Germany \\ ${ }^{4}$ Institut für Mineralogie, University of Münster, D-48149 Münster, Germany \\ ${ }^{5}$ ESRF-The European Synchrotron, CS 40220, 38043 Grenoble Cedex 9, France \\ ${ }^{6}$ AdlOptica Optical Systems GmbH, D-12489 Berlin, Germany
}

(Received 7 February 2017; accepted 31 July 2017; published online 22 August 2017)

\begin{abstract}
A portable double-sided pulsed laser heating system for diamond anvil cells has been developed that is able to stably produce laser pulses as short as a few microseconds with repetition frequencies up to $100 \mathrm{kHz}$. In situ temperature determination is possible by collecting and fitting the thermal radiation spectrum for a specific wavelength range (particularly, between $650 \mathrm{~nm}$ and $850 \mathrm{~nm}$ ) to the Planck radiation function. Surface temperature information can also be time-resolved by using a gated detector that is synchronized with the laser pulse modulation and space-resolved with the implementation of a multi-point thermal radiation collection technique. The system can be easily coupled with equipment at synchrotron facilities, particularly for nuclear resonance spectroscopy experiments. Examples of applications include investigations of high-pressure high-temperature behavior of iron oxides, both in house and at the European Synchrotron Radiation Facility using the synchrotron Mössbauer source and nuclear inelastic scattering. Published by AIP Publishing. [http://dx.doi.org/10.1063/1.4998985]
\end{abstract}

\section{INTRODUCTION}

Modern high-pressure research demands that properties of materials be studied in situ, for example, inside diamond anvil cells (DACs), ideally simultaneously at high temperature and high pressure. Among the two major methods of heating in DACs, namely, laser and resistive heating, ${ }^{1,2}$ laser heating has been already used for more than five decades ${ }^{3,4}$ and has numerous applications in mineral physics and high pressure chemistry, physics, Earth and material sciences. The laser heated diamond anvil cell (LHDAC) technique is used, for example, for simulating conditions similar to those in the deep Earth and planetary interiors or for investigating chemical processes and physical phenomena at extreme conditions. At synchrotron light source facilities, the LHDAC technique has been coupled with various experimental setups, such as Xray diffraction, ${ }^{5,6}$ Nuclear Inelastic Scattering (NIS), the Synchrotron Mössbauer Source (SMS), ${ }^{7,8}$ and X-ray Absorption Near Edge Structure spectroscopy (XANES). ${ }^{9,10}$

In addition to continuous-wave (CW) heating, pulsed lasers have been used starting from the first attempts to laser heat samples in a DAC, with the advantage of achieving significantly higher temperatures ${ }^{11,12}$ due to the concentration of the high laser power in a short impulse. Since the beginning of the 2000s, pulsed heating has been coupled with time-resolved measurements. ${ }^{13,14}$ The repetitive heating and cooling of the

\footnotetext{
a)Author to whom correspondence should be addressed: georgios.aprilis@ uni-bayreuth.de
}

sample makes time an extra variable in addition to pressure and temperature, which is not possible with continuous-wave laser heating. An entirely new field of research has been introduced, allowing determination of important material properties such as thermal conductivity and diffusivity and melting temperatures. ${ }^{15-18}$

The spatial distribution of temperatures at and around the heated spot is of great importance in a LHDAC experiment. The significance of a surface temperature map has been noted in many studies. ${ }^{19-24}$ Such a map is relevant especially to highpressure melting experiments ${ }^{21-24}$ but not exclusively. ${ }^{19,20}$ The methods used so far to determine the surface temperature distribution estimate the temperature profile along one line on the sample by leading the thermal emission into a spectrometer through a slit. ${ }^{21-23}$

In this work, we present a laser heating system in which a group of optical fibers probe the laser heated area at different spots. This solution provides a two-dimensional surface temperature map. To the authors' knowledge, there is so far no direct two-dimensional temperature mapping technique apart from computational estimations such as peak scaling ${ }^{20}$ or multispectral imaging radiometry approaches, in contrast to spectroradiometry. ${ }^{25}$ The use of optical fibers also allows us to physically separate the detector (and spectrometer) from the focusing optics and to avoid introducing additional optical components between them. These provide faster alignment and portability of the system that is especially important for synchrotron facilities, where the lack of space makes a free-space optical path solution unfeasible. ${ }^{19,26}$ Special care is taken for the laser beam shaping 
and improvement of the focusing optics in order to minimize the temperature estimation error caused by chromatic aberrations. $^{27,28}$

Thus, our portable pulsed laser heating system provides a solution for both temporally and spatially resolved temperature measurements in a double-sided LHDAC. It is designed to be easily movable between home institutions and synchrotrons or between different beam-lines, where the need for portability of laser heating systems has already been recognized. ${ }^{8,29-31} \mathrm{We}$ demonstrate its application through a series of experiments conducted at the Nuclear Resonance beamline (ID 18) of the European Synchrotron Radiation Facility (ESRF).

\section{SYSTEM OVERVIEW}

The system is shown as a general schematic in Fig. 1. The system consists of the following main components (described in more detail in the following sections):

- Two infrared lasers that independently heat a sample in the DAC from two sides.

- Two UniHead modules (by Precitec $\mathrm{GmbH}$ ) with optical components to shape and focus each laser beam.

- Laser modulation and synchronization units including photo-detectors, custom electronics, and an oscilloscope for control and visualization of the pulse shapes.

- Two VIS/NIR fiber bundles for collecting thermal radiation which are optically aligned on the sample.

- High-precision spectrometer coupled with a fast, intensified CCD detector used for time-resolved spectroradiometry measurements from both sides.

\section{A. Lasers}

We use two SPI RedPower R4 fiber lasers emitting at the near-infrared (NIR) central wavelength of $1070 \pm 10 \mathrm{~nm}-$ one with a maximal power of $100 \mathrm{~W}$ and the other with $50 \mathrm{~W}$. The lasers produce a randomly polarized Gaussian beam $\left(T E M_{00}\right)$ with a full width at $1 / e^{2}$ of $5 \pm 0.5 \mathrm{~mm}$. The lasers can be operated in both continuous-wave mode as well as modulated with maximum frequency up to $100 \mathrm{kHz}$. The minimum possible laser pulse width is on the order of a few microseconds, but a very short pulse increases the power control error of the feedback loop of the laser; therefore a hard limit of $5 \mu$ s is set by the system's modulation electronics to avoid power instabilities. Laser heating experiments can last up to many hours, and the data quality relies greatly on the laser power stability. In the system described here the laser manufacturer provides a power deviation of $<1.0 \%$ over $8 \mathrm{~h}$ for continuous-wave emission.

\section{B. Beam shaping and focusing}

The collimated output beam of the laser is very wide for the intended applications; therefore, it needs to be shaped and focused accordingly. Each laser beam is collected by a Focal- $\pi$ Shaper (Focal- $\pi$ Shaper_9_1064 by AdlOptica GmbH), which is designed to shape a $T E M_{00}$ beam into a flat-top when combined with focusing optics. ${ }^{32,33}$

The output beams of the Focal- $\pi$ Shapers from both sides are focused using two sets of GeoHEAT 40_NIR lenses [Fig. 2(a)]. GeoHEAT lenses are specially designed for laser heating applications with DACs. The required portability of the system prohibits the redundancy of optical paths; therefore, the optical elements for collection of thermal radiation are common with the ones used for laser heating. This setup demands focusing optics that can reliably operate in both spectral bands. GeoHEATs are achromatic objectives made to minimize chromatic aberration at the optimum spectral ranges of $600 \mathrm{~nm}-900 \mathrm{~nm}$ and $1020 \mathrm{~nm}-1100 \mathrm{~nm}$. The nominal chromatic focal shift for GeoHEAT 40_NIR can be seen in Fig. 3.

Samples under high pressure inside a DAC can have dimensions on the order of a few tens of micrometers; hence, in order to be able to create a distinct temperature spatial map, a physical resolution of $2.5 \mu \mathrm{m}$ is set as a specification. According to the Rayleigh criterion ${ }^{34}$ to provide a $2.5 \mu \mathrm{m}$ physical resolution, the diameter $d_{p}$ of the central spot of an Airy distribution in the focal plane of the lens has to be about $5 \mu \mathrm{m}$.

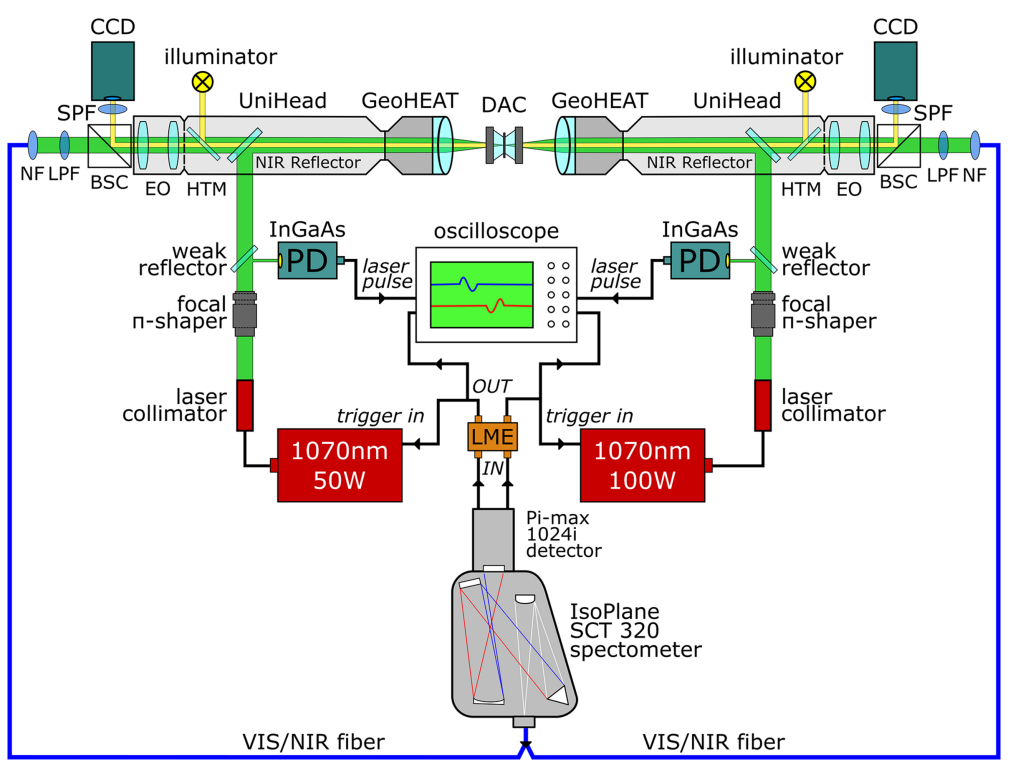

FIG. 1. Double-sided pulsed laser heating system for diamond anvil cells. SPFs are the short-pass filters with a cut-off at $850 \mathrm{~nm}$; CCDs are the cameras for sample visual observation; NFs are the notch filters at $1064 \mathrm{~nm}$ used to isolate the laser beam from the detector; LPFs are the longpass filters with a cut-on wavelength of $550 \mathrm{~nm}$ in order to avoid contamination from the second order diffraction of the grating; BSCs are the beam-splitter cubes; EOs are the "eyepiece" optics of the UniHead modules that focus the image on the viewing cameras and optical fibers; HTMs are the half-transparent mirrors that reflect illumination light; GeoHEATs are the achromatic lenses for laser focusing and collection of thermal radiation; PDs are the fast InGaAs photo-detectors used to capture laser pulses; and LMEs are the laser modulation electronics (the figure is not drawn to scale). 


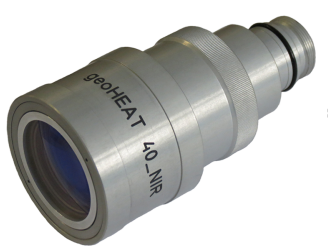

(a)

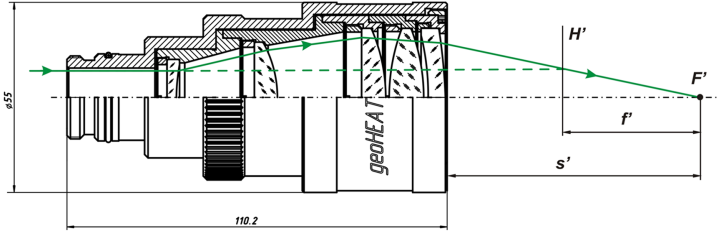

(b)
FIG. 2. (a) Photo and (b) optical layout of a geoHEAT $40 \_$NIR built as a reverse telephoto lens. $\mathrm{H}^{\prime}$ is the principle plane and $\mathrm{F}^{\prime}$ is the focal spot that defines the focal distance $\mathrm{f}^{\prime}$, that is shorter than $\mathrm{s}^{\prime}$, the working distance.
The f-number (or diaphragm number) of the lens $f / \#$ is calculated using the formula ${ }^{34}$

$$
f / \#=d_{p} /(2.44 \lambda) .
$$

For $\lambda=750 \mathrm{~nm}$, the center of the spectral band for measurements, the f-number results in $f / \#=2.7$. The GeoHEAT $40 \_$NIR is designed with $f / \#=2.6$ and the focal distance $f^{\prime}=40.8 \mathrm{~mm}$ (at $1064 \mathrm{~nm}$ ); thus, it meets the requirements. This combination provides a large enough field of view for optical sample alignment as well as a narrow depth of field that is necessary for precision in temperature measurements. The new optical setup is an improvement over previous designs ${ }^{8,31}$ by increasing the numerical aperture and magnification of the system and thus the resolution.

For simple objectives, the working distance is equal to the focal distance, but in the case of a portable system used for synchrotron applications, $40.8 \mathrm{~mm}$ is not enough. The space around a DAC should be larger and sufficient for inserting additional optical or X-ray optical elements, for example, carbon mirrors for synchrotron single-crystal X-ray diffraction studies in laser-heated DACs. ${ }^{8}$ The optical design of the GeoHEAT 40_NIR is implemented as a retrofocus (reverse telephoto) lens, as shown in Fig. 2(b). It is composed from two optical components (i.e., groups of lenses); the first one has a negative optical power, while the second one has a positive power. ${ }^{35} \mathrm{By}$ choosing optical powers of the components as well as an airgap between them, it is possible to fulfill the conditions of the required focal length and working distance.

The beam spot can have a radius as small as $20 \mu \mathrm{m}$, when a flat-top is used at the focusing distance. An even smaller size can be achieved by adjusting the Focal- $\pi$ Shaper, but with a Gaussian distribution. With a smooth transmission curve of $96 \%$ over the entire spectral range used for temperature

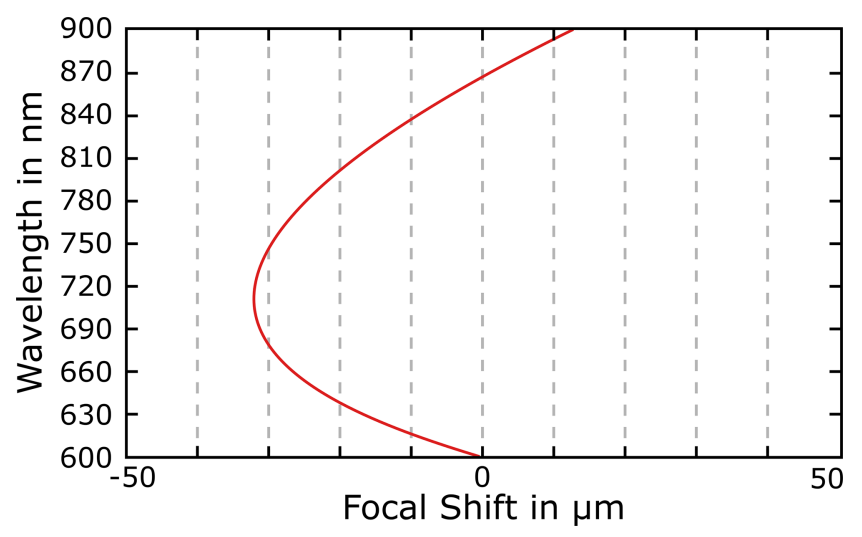

FIG. 3. Diagram of chromatic shift for geoHEAT 40_NIR. determination, the geoHEAT 40_NIR optics facilitate both spectroradiometry and laser heating of the sample, improving significantly the experimental conditions of LHDAC.

\section{Laser modulation and synchronization}

Synchronization of the lasers is crucial. In order to heat a sample as homogeneously as possible, the peak and duration of the laser pulses should match from both sides. Observation of the laser pulse is done by reflecting a small portion of the laser beam into a photodiode and then recording the response on the oscilloscope (Fig. 1). Two fast InGaAs detectors (DET08CL model from Thorlabs, Inc.) are used in this case, with rise and fall response times of less than a nanosecond. This allows an accurate capturing of the shape of short pulses lasting only a few microseconds.

The main trigger of the system comes directly from the internal trigger generator of the PI-MAX detector. The detector's generator provides a set of two triggers (named SyncMaster1 and SyncMaster2) with a common frequency and a relative delay with respect to each other that can be set by software. These two outputs are led to a custom electronics module that converts the fixed-width triggers into modulation pulses with adjustable width that control the laser behavior.

The modulation electronics are depicted in Fig. 4. The two inputs have a fixed width of 500 ns. Two timer circuit modules connected as monostable multivibrators can shape the incoming triggers into square modulation pulses. The width of the output pulses can be manually set with the help of an oscilloscope and two variable resistors.

In this way, it is possible to easily control the common modulation frequency of the lasers, the duty cycle of their emission independently from each other, and their relative delay. The latter is important since the response time of the laser depends strongly on the working conditions, such as the duty cycle and the emitting power; thus it is likely that the two lasers will not respond in exactly the same way, even if they receive their trigger at the same time.

An example of an oscilloscope screen is shown in Fig. 5. A relative delay between two laser triggers and modulation pulse width can be easily adjusted using the screen as a reference, in order to match the laser peak position and pulse width. Their synchronization can be finely tuned with a precision on the order of a few nanoseconds, and it is possible to investigate the thermal response of the sample, especially in the area around the laser peaks. This usually appears at the beginning of the pulse, when even a very small delay between the laser impulses can strongly affect the sample's thermal response between the two heated sides. ${ }^{36}$ 


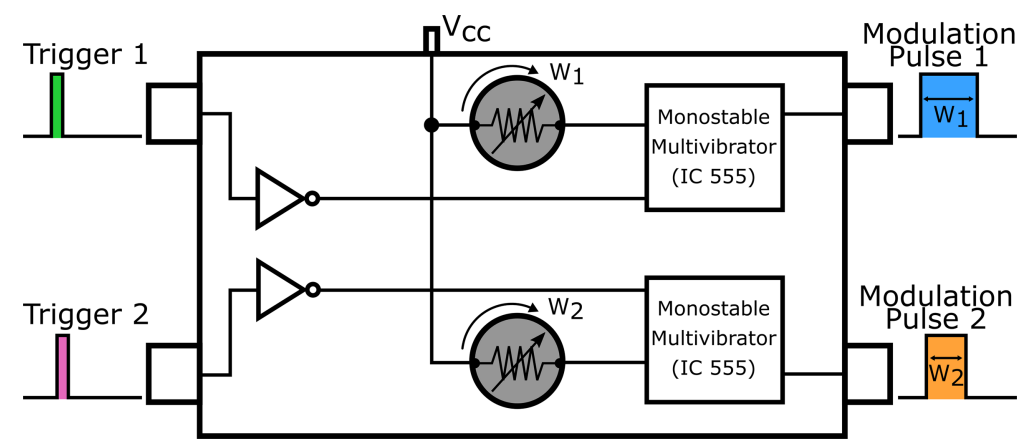

FIG. 4. Schematic of the electronics module that controls the laser modulation. The two input triggers are generated by the PI-MAX detector with fixed width and have adjustable relative delay. Two square pulse outputs independently modulate the two lasers in the pulsed mode.

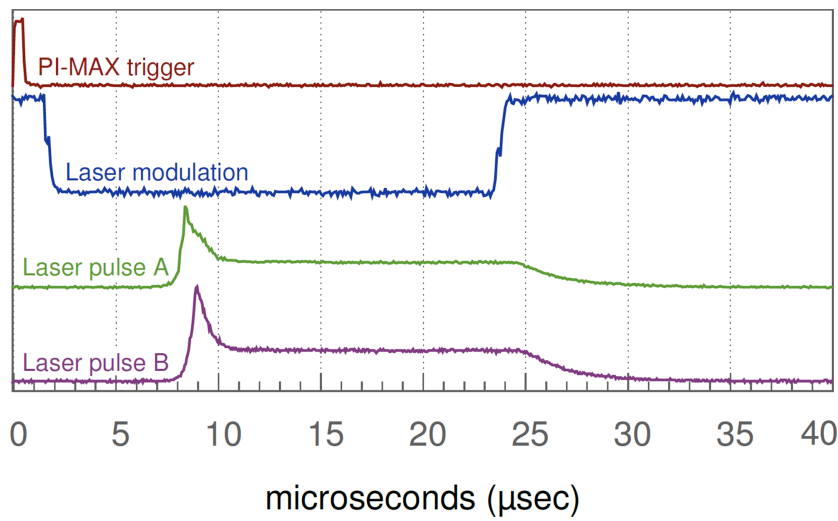

FIG. 5. Example of an oscilloscope screen. PI-MAX sets the frequency by generating short triggers. The lasers are modulated with negative logic, i.e., the laser is ON when the voltage is LOW. This figure demonstrates the signals needed to create laser pulse A. A similar set of PI-MAX trigger and modulation signals are also sent to the second laser, creating the laser pulse B.

\section{Collection of thermal radiation and temperature determination}

The accurate in situ determination of the temperature of the laser-heated sample inside the DAC is crucial. The optical transparency of the diamond anvils allows the collection of the emitted radiation of the heated sample and the estimation of its temperature using the gray body approximation of Planck's law (Fig. 6). ${ }^{37}$ The thermal radiation is collected from both sides through the same optical path that is used to focus the laser. This is done with a set of two
UniHead modules, in order to maintain the portability of the system.

The image of the sample inside the DAC is magnified by the UniHead optics, working as a microscope, and projected into the cameras that are used for optical observation. A proportion of this light is collected by a pair of fiber bundles using a pair of 50-50 beam-splitter cubes (Fig. 1). The core size and the number of fibers of the bundle can vary according to the experimental needs in order to change the spatial alignment relative to the heated sample and the amount of collected heat radiation. The eyepiece optics are a combination of lenses, designed and installed by Precitec $\mathrm{GmbH},{ }^{8}$ which allow a working distance of $150 \mathrm{~mm}$ from the last lens surface to the fiber, providing a total of $20 \times$ magnification to the system, when used in combination with the geoHEAT 40_NIR focusing lenses.

A notch filter with a center wavelength of $1064 \mathrm{~nm}$ is placed right in front of the fiber bundles on each collection side to isolate them from any reflected laser radiation (Fig. 1). Similarly, longpass filters with cut-on wavelengths of $550 \mathrm{~nm}$ are used to avoid contamination from the second order diffraction of the grating, especially during the measurement of very high temperatures (above $4000 \mathrm{~K}$ ) when the intensity of radiation with wavelengths shorter than $500 \mathrm{~nm}$ is significant. ${ }^{38}$

The light is guided into an IsoPlane SCT 320 spectrometer (Princeton Instruments) and the resulting spectrum is collected by a PI-MAX 4 with a $1024 \times 256$ ICCD detector. The Intensified CCD camera with enhanced quantum efficiency gives the possibility of very fast collection. It can work on the gated

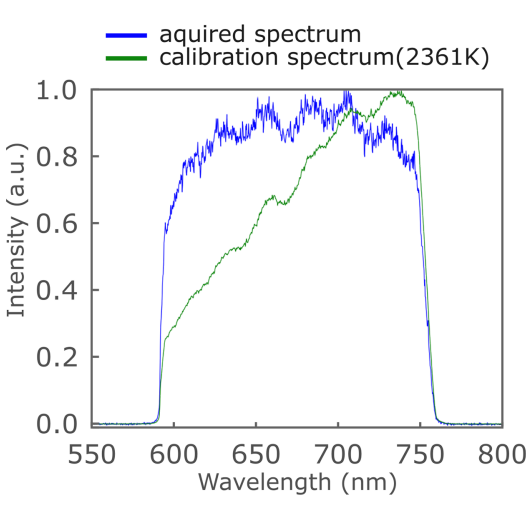

(a)

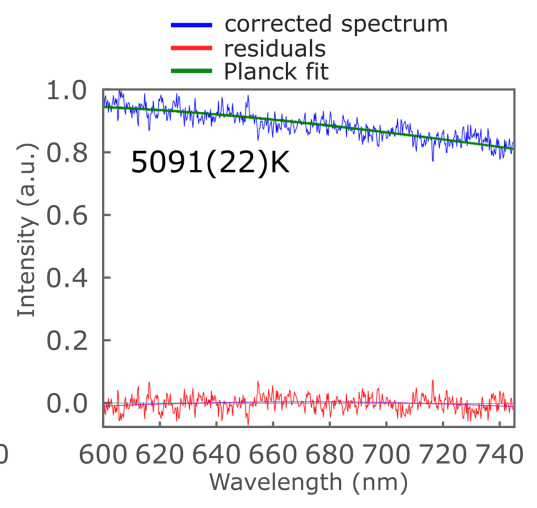

(b)
FIG. 6. Example of a successful temperature determination of heated $\varepsilon-\mathrm{O}_{2}$ at $46 \mathrm{GPa}$ inside a DAC using the gray body approximation of Planck's law: (a) calibration and acquired spectra; (b) fitting using gray body approximation. 
mode, which allows time-resolved collection of the emitted thermal radiation.

The exposure window during which a thermal radiation spectrum is collected in the current set-up can be as short as $\approx 4$ ns. Moreover, delay increments down to $0.1 \mathrm{~ns}$ can be selected, with the possibility to create a time-resolved temperature series with overlapping exposure windows. For a given spectrum, a minimum amount of counts is necessary in order to overcome the background noise and successfully determine the temperature. Therefore, whereas optical spectra may in principle be collected from every individual laser pulse, usually each gated window of the detector is collected over many repetitions, some hundreds or thousands of pulses accumulating during the total exposure time. For this reason, it is crucial that the laser heating is sufficiently stable, i.e., that all pulses have identical shape and cause the same effect on the sample.

In order to create a time series of temperatures along the duration of the laser pulse (Fig. 7), it is crucial to correlate the laser pulse period with the gated exposure windows of the camera. A common modulation trigger is used for the lasers and the spectrometer camera signifying the beginning of each

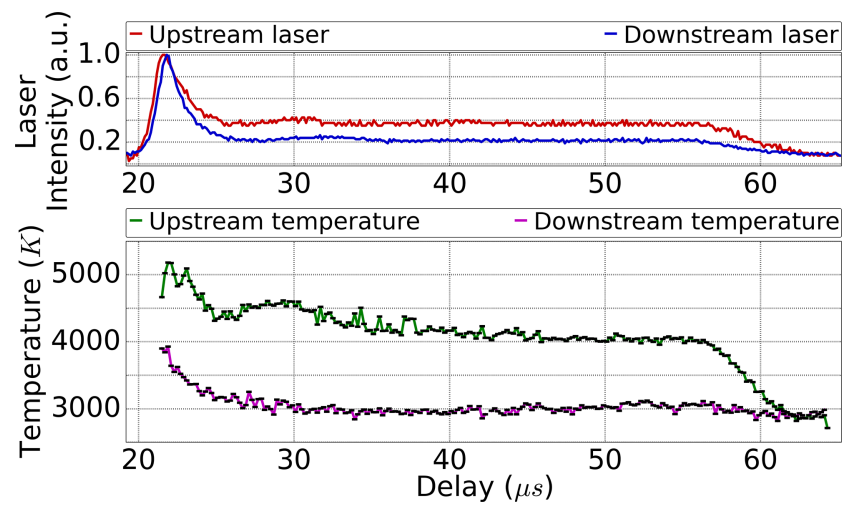

FIG. 7. Temperature measured during double-sided pulsed laser heating of solid $\varepsilon-\mathrm{O}_{2}$ inside a DAC at $46 \mathrm{GPa}$ with $10 \mathrm{kHz}$ repetition rate. For the purpose of this experiment, the laser pulse width was set to approximately $40 \%$ duty cycle, i.e., a duration of $40 \mu$ s. The $100 \mu$ s pulse period is divided into windows of $200 \mathrm{~ns}$ each. The figure includes the part of the period when the heat radiation was sufficiently high to be detected. The collection windows are denoted by black horizontal lines and the solid colored lines are the guides for the eye. pulse period, created internally by the pulse generator of the PI-MAX detector. The laser response and trigger signal are observed using an oscilloscope as described in Sec. II C. Using the trigger signal as a time reference point, the arrival of the laser pulse relative to the trigger can be correlated with the relative delay of each gated exposure window of the camera, making it possible to determine the time-resolved temperature response of the sample to laser heating.

When working in the nanosecond timing range, cable and equipment delays become significant. Hence, special care is taken to correct the temperature-laser synchronization by taking into account the response delays of cables and electronics. Using the internal trigger $T_{0}$ of the PI-MAX detector as a time reference point, all cable and equipment delays are recorded on the oscilloscope and subtracted to give an absolute timestamp to every event. The delay of the photodiodes and their cables is calculated using the spectrometer to capture the real laser pulse (attenuated significantly). A time-resolved collection gives the absolute laser pulse delay which is then compared with the delay of the laser pulse recorded using the oscilloscope and appropriately corrected.

\section{E. Temperature distribution in the heated spot}

The time-resolved temperature measurement technique, when combined with multiple temperature acquisitions from a multi-core fiber bundle, results in a two-dimensional map of surface temperatures that resolve the sample thermal response both spatially and temporally. This method can potentially reveal much information regarding the thermal dynamics of the sample and accurately determine the temperature of the sample within the heated spot.

In our system, a set of 30 fiber cores is used, divided into two groups of 15 fibers each, in fiber bundles that are aligned optically to the center of the laser heating area from both sides of the DAC. The fibers have a diameter of $100 \mu \mathrm{m}$ and are grouped in two circular clusters [Fig. 8(a)], one from each side, but there is flexibility to switch between different fiber sets, depending on the desired application. The actual temperature probing diameter of each fiber depends on the fiber core size as well as on the optical focus and magnification. This can be easily determined during the alignment process by shining light into the fibers as well as on a reflective surface

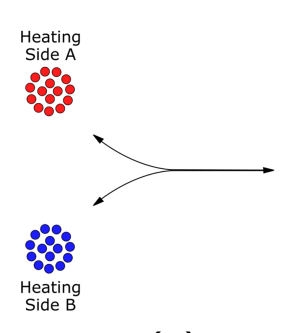

(a)
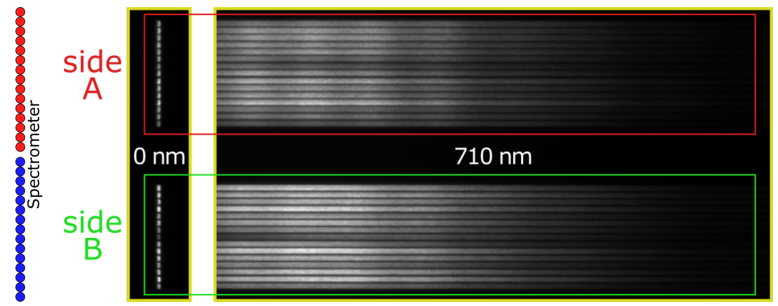

(b)

FIG. 8. (a) The bifurcated fiber optic bundle used for spectroradiometry measurements consists of two bundles with 15 fiber cores of $100 \mu$ m each, aligned in a "bunch" formation at the collection side. At the spectrometer side, the alignment is linear, with both legs joined at the same ferrule. (b) Portions of the detector images showing the signal from all fibers when both sides are focused on an illuminated platinum foil, at two grating positions. Left: grating is at "zero position," i.e., the detector is aligned with its zero order diffraction. Right: the center position of the grating is at $710 \mathrm{~nm}$, showing the dispersion of the illumination light. 
that is placed at the DAC position looking at the actual lit area. Typical values for a $400 \mu \mathrm{m}$ core fiber patch cable are approximately $20-25 \mu \mathrm{m}$.

At the spectrometer side, both fiber bundles are aligned linearly, one on top of the other, so that each thermal radiation spectrum lies on a different area of the detector. In the case of PI-MAX $41024 \times 256$, the pixel size is $26 \mu \mathrm{m} \times 26 \mu \mathrm{m}$, which defines the limits of fiber core size and inter-fiber spacing. Each region of interest of the detector can be correlated with a spot on the heated sample that has a specific diameter and position, and it is possible to create a two dimensional surface temperature map for each side of the sample [Fig. 8(b)]. Examples of applications are presented in Sec. III.

The fast alignment process allows the use of different temperature collection set-ups by simply exchanging and adjusting the fibers without requiring a realignment of the entire optical path. This feature is a useful capability of the system, especially when used during a synchrotron experiment where time is usually limited and therefore precious.

\section{EXAMPLES OF APPLICATION}

Some examples of successful application of the developed system are presented in Secs. III A-III C and demonstrate the capabilities of the system. Two-dimensional temperature mapping is shown for an in house experiment on pulsed laser heating of an $\mathrm{Fe}_{2} \mathrm{O}_{3}$ crystal at high pressure. The stability and portability of the system is demonstrated in a series of experiments using Synchrotron Mössbauer Source spectroscopy 39 and Nuclear Inelastic Scattering (NIS) spectroscopy coupled with pulsed LHDAC at the Nuclear Resonance (ID18) beamline ${ }^{40,41}$ of the European Synchrotron Radiation Facility (ESRF).

For the experiment, the system was transported to ESRF and mounted on the experimental hutch of the Nuclear Resonance beamline. The entire procedure of connecting and aligning usually requires only $4-6 \mathrm{~h}$ at the beginning of an experiment. It may be easily performed during a shut down or maintenance day so that precious experimental time lost to preparation is kept to a minimum.

In order to allow the X-ray beam to probe the sample along the compression axis of the DAC, it is necessary to partially modify the geometry of the system. In the case of NIS experiments, the laser heads are placed in the same co-axial orientation and perpendicular to the X-ray beam, while the DAC compression axis coincides with the direction of the beam. The sample is heated from both sides, "on-axis" with the X-rays, using two carbon mirrors positioned at $\approx 45^{\circ}$ angles in front of the geoHEAT 40_NIR focusing optics, similar to the setup in Ref. 36. While X-ray absorption of the carbon mirrors is low, it is a crucial consideration for SMS experiments when the beam intensity is weak. The carbon mirrors are therefore replaced by a pair of D-shaped, silver-coated glass mirrors at $\approx 42^{\circ}$ angles from the focusing optics. In this way, the incident X-ray beam is allowed to pass without going through the mirrors and the heating laser beam is as close to the DAC compression axis as possible.

\section{A. Time-space resolved measurements}

In a series of in house experiments, we acquired both timeand space-resolved surface temperature data for an $\mathrm{Fe}_{2} \mathrm{O}_{3}$ crystal inside a DAC. The sample was loaded into the pressure chamber (Re gasket indented to $27 \mu \mathrm{m}$ with a $100 \mu \mathrm{m}$ diameter hole mounted on a DAC with diamond anvils of $150 \mu \mathrm{m}$ culet size) with $\mathrm{Ne}$ as both a pressure transmitting medium and thermal insulator. The pressure chamber was compressed to $106 \mathrm{GPa}$ and heated from one side (hereafter "Side B") with laser pulses of $31.5 \mathrm{~W}$ power using a defocused beam. The pulse duration was $14 \mu \mathrm{s}$ with a frequency of $25 \mathrm{kHz}$, resulting in a $40 \%$ duty cycle. The heating spot was aligned to be roughly at the center of the observation area of the fiber cluster. As seen in Fig. 9, it was possible to detect the propagation of heat and measure temperature at one side of the sample during pulsed heating on the opposite side. The temperature initially increases at the heated side during the first moments of laser heating at time point "A," and right after the peak of the pulse at time point " $\mathrm{B}$," detectable temperature appears also at the center of the non-heated side, being some hundreds of degrees lower. At time point "C," a few microseconds after the pulse peak, the non-heated side cools down to undetectable temperatures when the temperature on the heated side is not high enough.

Within the heated spot, which in this case is only the central part of the probing area, it is possible to detect a temperature gradient of more than $200 \mathrm{~K}$ over a distance of $15-20 \mu \mathrm{m}$. This demonstrates the importance of having a flat laser beam to avoid steep temperature gradients, especially when temperature is determined by probing a relatively large area.

It is worth mentioning that temperatures at the non-heated side sometimes appear to be slightly higher than on the heated side, as in the example of Fig. 9 at time point "B." Such an observation can be due to individual fibers not being exactly aligned on the same spot on each side of the sample, where there can be a distance of a few micrometers between the observation spots, each having a slightly different temperature. Moreover, temperature differences of a few tens of degrees Kelvin are within the detection uncertainty, which is generally accepted to be on the order of one hundred degrees for laser heating experiments.

\section{B. Synchrotron Mössbauer source at the ESRF}

Time-resolved SMS absorption spectra can be collected during pulsed heating of the sample by combining the pulsed laser heating system with newly developed beamline electronics. In this way, the pulsed heating period can be "sliced" into time windows of specified width and the Mössbauer absorption of the sample, as well as its surface temperature corresponding to each time window can be calculated accordingly. The system cabling and assembly are shown in Fig. 10 as well as the delay for each signal path, which is necessary for the correct synchronization of the data. The photodiode signal timing was measured by directly capturing the laser pulse using the spectrometer and comparing it with the photodiode response. All other delays were measured using a direct comparison of the signals on the oscilloscope. The time-resolved SMS setup including synchrotron 
SIDE A

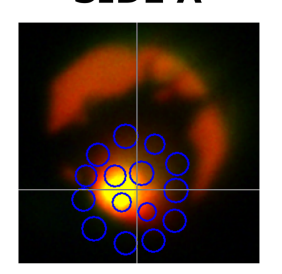

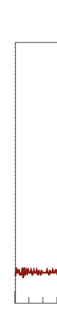
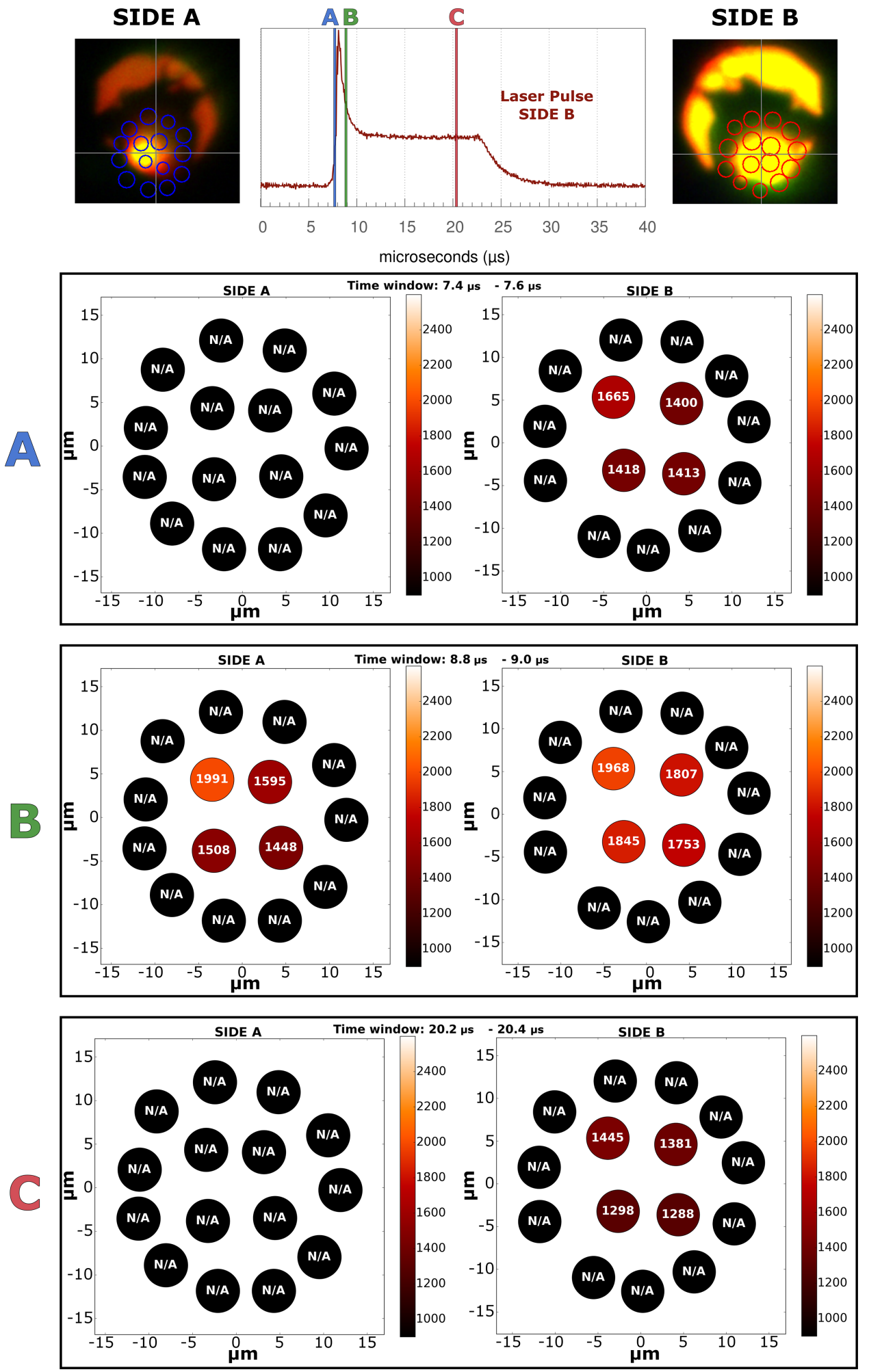

FIG. 9. Time dependence of the laser pulse (red). Vertical blue, green, and red bars indicate $200 \mu$ s time windows starting, respectively, at $7.4,8.8$, and $20.2 \mu$ s after the spectrometer trigger Left and right insets show an image of two heating sides. Parts (A)-(C) show the time-resolved temperature maps of $\mathrm{Fe}_{2} \mathrm{O}_{3}$ obtained at $106 \mathrm{GPa}$ using onesided pulsed heating of $31.5 \mathrm{~W}$ pulse power and frequency of $f=25 \mathrm{kHz}$. The temperature induced at the back (nonheated side) of the sample can be seen close to the peak of the pulse at time window "B." Temperatures noted as "N/A" denote temperature values that are too low to be estimated using spectroradiometry, usually below $1100 \mathrm{~K}$. electronics and data treatment is beyond the scope of this publication and will be described in detail elsewhere as a separate system.

An iron foil enriched in ${ }^{57} \mathrm{Fe}$ was placed into a DAC using paraffin as a pressure transmitting medium and pressurized to $64 \mathrm{GPa}$, with the goal to form iron hydride $\mathrm{FeH}_{\mathrm{x}}$ upon heating. First, the sample was heated continuously for several minutes at a maximum temperature of $1500 \mathrm{~K}$ from both sides. Then, it was double-sided pulse heated at $20 \mathrm{kHz}$ frequency.
The two laser pulses were synchronized and both had lengths of approximately $20 \mu \mathrm{m}$, i.e., around $50 \%$ duty cycle, and both were at a power of $P \approx 10 \mathrm{~W}$ at the pulse plateau. Temperature was estimated with spectroradiometry using two single-core fiber patch cables with cores of $400 \mu \mathrm{m}$ diameter, collecting light from an area of approximately $20 \mu \mathrm{m}$ in diameter that was centered at the heating spot. The laser pulses, as well as the sample temperature response with $1 \mu$ s temporal resolution, are presented in Fig. 11(c). The mean value of a series of 


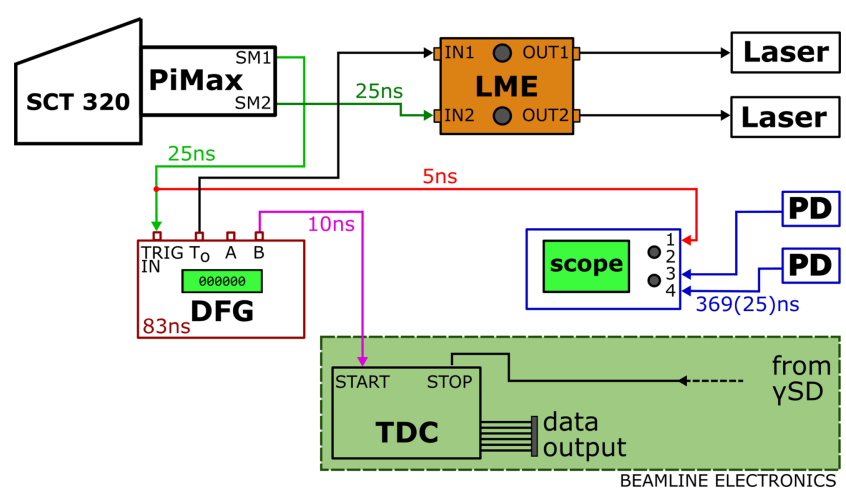

FIG. 10. System layout and signal paths for time-resolved SMS experiments with pulsed laser heating. SCT 320 and PiMax are the spectrometer and detector, respectively, the latter producing two triggering pulses for the lasers; LME are the laser modulation electronics; DFG is a digital function generator used to signal the beamline electronics at the beginning of each pulse period; scope is the oscilloscope for observing the laser pulses; PD are the InGaAs photodiodes; TDC is a time-to-digital converter that collects and treats the time-resolved SMS data; and $\gamma \mathrm{SD}$ is the gamma scintillation detector used in the SMS setup.

data collections is shown, along with one standard deviation (STD) margins. The heating run lasted around $30 \mathrm{~min}$ with temperature collection approximately every $5 \mathrm{~min}$.

The centre shift (CS) of a Mössbauer absorption line is linearly dependent on temperature, ${ }^{42}$ so the temperature of the entire probed volume of the sample can be estimated from the difference of the $\mathrm{CS}$ of the component(s) relative to the CS of the same component(s) at a known temperature. The sample is coldest during the heating cycle at the moment right before the peak of the pulse. The actual temperature during cooling depends on the properties of the sample and its environment, as well as on heating conditions such as duty cycle, power, and frequency. It is expected to be close to room temperature compared with the temperatures developed during heating. In this way, Mössbauer spectroscopy provides an estimation of the variation in sample temperature at different moments of heating.

The SMS absorption spectrum corresponding to 6.5-8.5 $\mu$ s delay from the beginning of the pulse period, right before the laser pulse, is shown in Fig. 11(a). The centre shift is $0.42(3) \mathrm{mm} / \mathrm{s}$ based on a fit using MossA software. ${ }^{43}$ Figure 11(b) shows the SMS absorption spectrum collected at a time window of $23-25 \mu$ s that has a CS of $-0.39(6) \mathrm{mm} / \mathrm{s}$. Using the relation described in Ref. 42, the sample in this time interval is $1110(89) \mathrm{K}$ hotter than right before the arrival of the laser pulse. Considering that the surface temperature during the hot period is estimated to be approximately $1700 \mathrm{~K}$ on both sides of the sample, cooling to around $600 \mathrm{~K}$ takes place right before the next pulse arrives.

\section{Nuclear inelastic scattering at ESRF}

Nuclear inelastic scattering requires very long data acquisition time in order to obtain sufficient statistics for meaningful

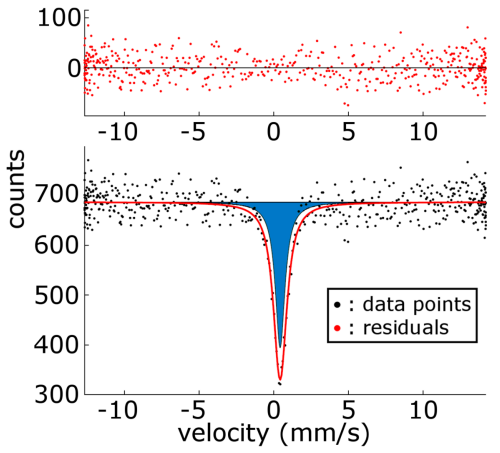

(a)

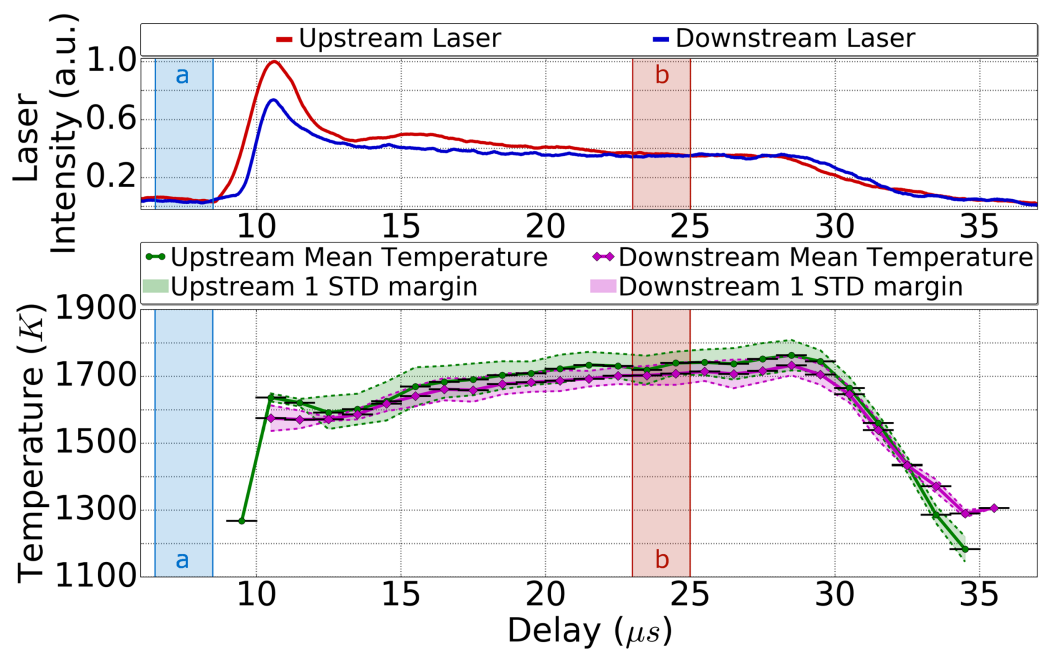

FIG. 11. Mössbauer absorption spectra of $\mathrm{FeH}_{\mathrm{x}}$ at $64 \mathrm{GPa}$ corresponding to cooled [(a), right before pulse, $6.5-8.5 \mu$ s delay from the beginning of the pulse period] and heated sample [(b), collected at the time window 23-25 $\mu \mathrm{s}$ ]. (c) Mean temperatures along the pulse as determined from spectroradiometric measurement (with one standard deviation margins) compared with the laser pulse profiles for upstream and downstream. The shaded time windows correspond to the cold and hot spectra from (a) and (b). 
results. A single energy scan can last from half an hour to one hour, and many repetitions are necessary to improve statistics, resulting in a collection time of many hours. In the case of NIS experiment with a pulsed laser heated DAC, the main challenge is to maintain the stability of heating over time. Every pulse should produce the same temperature response on the sample or at least the difference over time should be traceable to any possible correlations with experimental conditions (e.g., chemical reaction of the sample).

An iron foil enriched with ${ }^{57} \mathrm{Fe}$ was loaded into a DAC of $250 \mu \mathrm{m}$ culet size, with $\mathrm{Al}_{2} \mathrm{O}_{3}$ as a pressure transmitting medium as well as a thermal insulator. The sample was pressurized to $30 \mathrm{GPa}$, taking extra care to insulate the sample from the diamond anvils that act as good thermal conductors. The sample was laser heated from both sides using pulses of $\approx 20 \mu$ s duration, a frequency of $16 \mathrm{kHz}$ (i.e., $32 \%$ duty cycle) and pulse powers at the pulse plateau of $P_{u p}=14.6 \mathrm{~W}$ and $P_{\text {down }}=15.1 \mathrm{~W}$ for upstream and downstream heating sides, respectively. The surface temperature of the heated sample was estimated with spectroradiometry, using two fiber patch cables with $400 \mu \mathrm{m}$ core diameter that were focused on an area of approximately $20 \mu \mathrm{m}$ around the center of the laser heated spot. The run duration of the temperatures presented here lasted $\approx 12 \mathrm{~h}$ while maintaining a stable laser heating setup. In total, 54 temperature time-series were collected with $\approx 15$ min intervals between them. Each window of the time-series is $500 \mathrm{~ns}$ long and was collected from a repetition of a few tens of thousands of pulses, as described in Sec. II D. This resulted in $\approx 3$ min of total acquisition time for each dataset. The pulse shapes and power of the two lasers were manually adjusted to be synchronized and result in a similar surface temperature on both sides of the Fe sample.

The mean temperature for each time window is depicted in Figs. 12(a) and 12(b) for upstream and downstream sides, respectively, including the areas of \pm 1 standard deviation (STD) over a total of 54 collections. The standard deviation was around $100 \mathrm{~K}$ at the flat part (plateau) of the pulse and $300 \mathrm{~K}$ at the peak position, close to the uncertainty level of spectroradiometry measurements.

The heated sample was probed with an X-ray beam size of $12 \mu \mathrm{m} \times 7 \mu \mathrm{m}$ and the energy dependence of NIS for Fe was collected. Details of the time-resolved technique for NIS measurements will be published elsewhere. Time windows can be selected along the period of the pulse and the corresponding scattering spectra can be calculated, tracking the behavior of the sample during each heating cycle. For demonstration purposes, three time windows were selected: before the pulse, after the peak, and after the drop of the pulse, as shown in Fig. 12. NIS can be used to determine the mean temperature of the sample volume that is probed by the X-ray beam, along with the surface temperature provided by spectroradiometry. The probability ratio between phonon creation and phonon annihilation for a given energy E, i.e., the symmetry of the spectrum around the elastic line, is a function of temperature. ${ }^{7,36,40}$ In Fig. 12(c), the estimated temperatures for each time window can be seen, where the phonon creation/annihilation ratio is calculated using the energy range of 10-20 meV (shaded areas). Before and after the laser pulse, the temperatures are low hence not detectable using

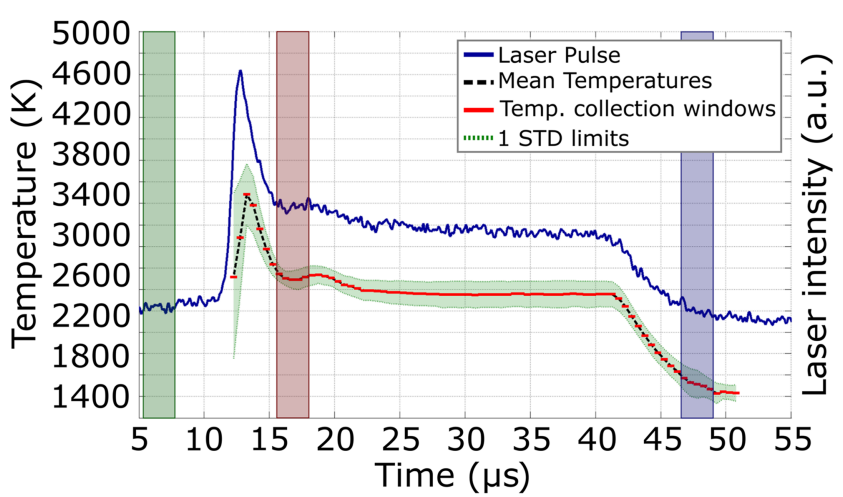

(a)

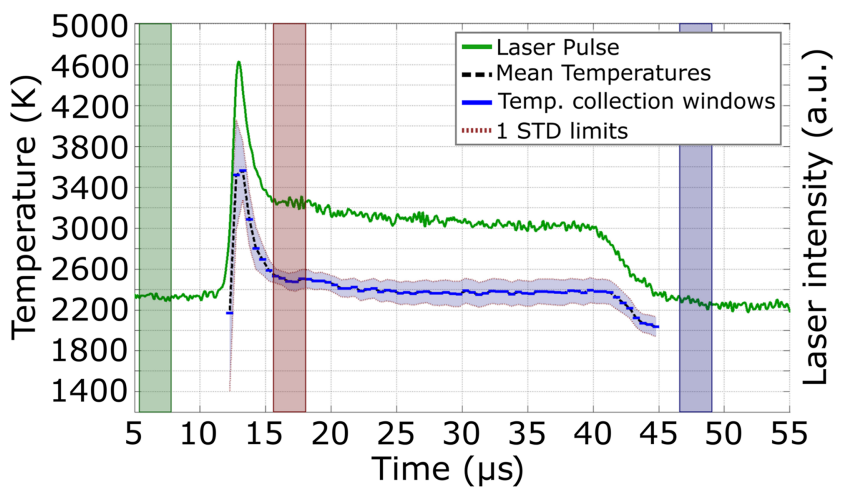

(b)

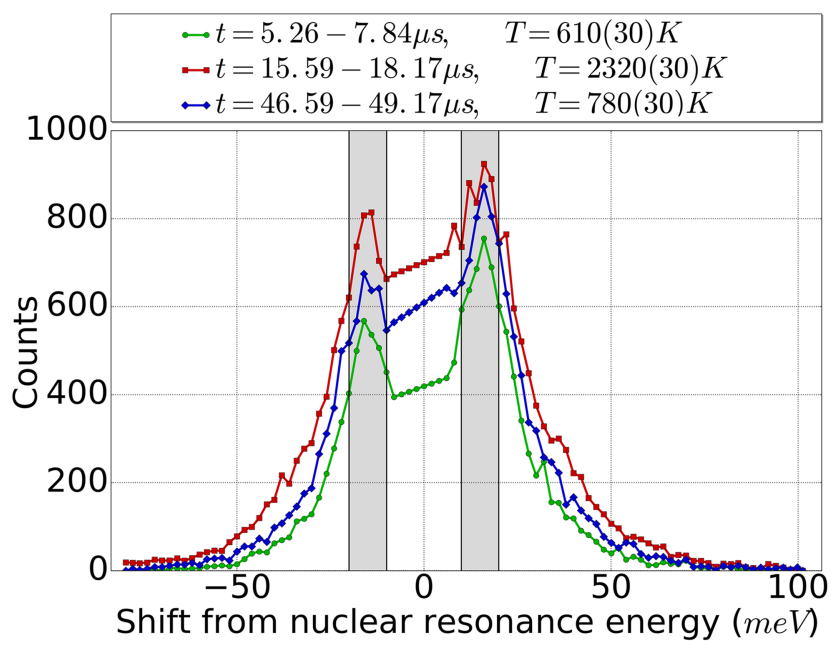

(c)

FIG. 12. (a) Upstream and (b) Downstream side mean surface temperatures and their standard deviations for the entire duration of data collection. Three different time windows are highlighted that correspond to the NIS spectra. (c) Energy dependence of nuclear inelastic scattering (elastic component removed) of ${ }^{57} \mathrm{Fe}$ for three different temporal positions along the pulse period. Temperatures are determined using the detailed balance law.

spectroradiometry at the visible light wavelength range. Nevertheless, the sample is still approximately $300^{\circ}-400^{\circ}$ above room temperature even when the laser is not emitting. During the pulse, the mean temperature in the heated spot appears to be around $2320 \mathrm{~K}$, which is $200^{\circ}-400^{\circ}$ lower than the surface temperatures on both sides of the sample. This is good agreement if we consider the expected temperature gradients between the surface and the center of the sample. 


\section{CONCLUSIONS}

A double-sided pulsed laser heating system was developed that is able to acquire time-resolved and space-resolved surface temperature information of samples under high pressure inside a diamond anvil cell, with temporal and spatial resolution of a few nanoseconds and micrometers, respectively. The portability and stability of the system were demonstrated, as well as the possibility to couple it with various techniques such as nuclear inelastic scattering at a synchrotron light source facility.

Special care was taken to minimize the chromatic aberration of the system, which is a known problem in laser heating setups with spectroradiometry temperature determination, and to keep the two laser pulses independent with respect to the power, delay, and duration, in order to maximize experimental flexibility.

The pulsed laser heating system can be successfully used for time-resolved studies and coupled with different spectroscopic techniques. This allows the in situ investigation of transitional phenomena, including the determination of thermal conductivity, melting curve, and rheological properties of materials of geological and materials science significance at conditions of the Earth's interior. Heating with pulses can potentially provide solutions for the study of materials in cases that were difficult with continuous heating. Materials such as hydrogen and hydrides have a high risk of destroying diamond anvils, and other materials can react with them during persistent heating, forming carbides. In many of these cases, pulsed laser heating can minimize the heating time during measurements and prevent chemical interactions. The portable laser heating system can be a versatile tool, employed in a wide range of different applications.

\section{ACKNOWLEDGMENTS}

We acknowledge the European Synchrotron Radiation Facility (ESRF) for provision of beam time at ID18. N.D. thanks the German Research Foundation [Deutsche Forschungsgemeinschaft (DFG)] for financial support through the DFG Heisenberg Programme and Project Nos. DU 9548/1 and DU 954-11/1, and the Federal Ministry of Education and Research (BMBF; Germany) for BMBF Grant Nos. 5K13WC3 (Verbundprojekt O5K2013, Teilprojekt 2, PTDESY) and 5K16WC1. L.D. thanks the DFG and the BMBF (Germany) for Grant Nos. DU 393/10-1 and DU 393/10-1. The project was also supported by funds from the DFG through the CarboPaT Research Unit No. FOR2125 (Mc 3/20, Du 393/9).

${ }^{1}$ M. I. Eremets, High Pressure Experimental Methods (Oxford University Press, 1996).

${ }^{2}$ N. Dubrovinskaia and L. Dubrovinsky, Advances in High-Pressure Technology for Geophysical Applications (Elsevier, New York, 2005), p. 487. ${ }^{3}$ W. A. Bassett, Rev. Sci. Instrum. 72, 1270 (2001).

${ }^{4}$ W. A. Bassett, Diamond Anvil Cells: Laser Heating of Samples at High Pressure: 50 Years (Laser Focus World, 2016).

${ }^{5}$ E. Schultz, M. Mezouar, W. Crichton, S. Bauchau, G. Blattmann, D. Andrault, G. Fiquet, R. Boehler, N. Rambert, B. Sitaud, and P. Loubeyre, High Pressure Res. 25, 71 (2005).

${ }^{6}$ Y. Meng, R. Hrubiak, E. Rod, R. Boehler, and G. Shen, Rev. Sci. Instrum. 86, 072201 (2015).
${ }^{7}$ J. F. Lin, W. Sturhahn, J. Zhao, G. Shen, H. K. Mao, and R. J. Hemley, Geophys. Res. Lett. 31, L14611, doi:10.1029/2004GL020599 (2004).

${ }^{8}$ I. Kupenko, L. Dubrovinsky, N. Dubrovinskaia, C. McCammon, K. Glazyrin, E. Bykova, T. Boffa Ballaran, R. Sinmyo, A. I. Chumakov, V. Potapkin, A. Kantor, R. Rüffer, M. Hanfland, W. Crichton, and M. Merlini, Rev. Sci. Instrum. 83, 124501 (2012).

${ }^{9}$ G. Aquilanti, S. Pascarelli, O. Mathon, M. Muñoz, O. Narygina, and L. Dubrovinsky, J. Synchrotron Radiat. 16, 376 (2009).

${ }^{10}$ C. Marini, I. Kantor, O. Mathon, and S. Pascarelli, High Pressure Res. 33, 108 (2013).

${ }^{11}$ W. A. Bassett and L. C. Ming, Phys. Earth Planet. Inter. 6, 154 (1972).

${ }^{12}$ J. S. Gold, W. A. Bassett, M. S. Weathers, and J. M. Bird, Science 225, 921 (1984).

${ }^{13}$ N. Funamori and T. Sato, Rev. Sci. Instrum. 77, 093903 (2006).

${ }^{14}$ A. F. Goncharov and J. C. Crowhurst, Rev. Sci. Instrum. 76, 063905 (2005).

${ }^{15}$ R. S. McWilliams, D. A. Dalton, Z. Konôpková, M. F. Mahmood, and A. F. Goncharov, Proc. Natl. Acad. Sci. U. S. A. 112, 7925 (2015).

${ }^{16}$ L. Yang, A. Karandikar, and R. Boehler, Rev. Sci. Instrum. 83, 063905 (2012).

${ }^{17}$ A. F. Goncharov, M. Wong, D. A. Dalton, J. G. O. Ojwang, V. V. Struzhkin, Z. Konôpková, and P. Lazor, J. Appl. Phys. 111, 112609 (2012).

${ }^{18}$ P. Beck, A. F. Goncharov, V. V. Struzhkin, B. Militzer, H. K. Mao, and R. J. Hemley, Appl. Phys. Lett. 91, 181914 (2007).

${ }^{19}$ R. Sinmyo and K. Hirose, Phys. Earth Planet. Inter. 180, 172 (2010).

${ }^{20}$ E. S. G. Rainey and A. Kavner, J. Geophys. Res.: Solid Earth 119, 8154, doi:10.1002/2014jb011267 (2014).

${ }^{21}$ A. R. Thomson, M. J. Walter, O. T. Lord, and S. C. Kohn, Am. Mineral. 99, 1544 (2014)

${ }^{22}$ O. T. Lord, E. T. H. Wann, S. A. Hunt, A. M. Walker, J. Santangeli, M. J. Walter, D. P. Dobson, I. G. Wood, L. Vočadlo, G. Morard, and M. Mezouar, Phys. Earth Planet. Inter. 233, 13 (2014).

${ }^{23}$ O. T. Lord, M. J. Walter, R. Dasgupta, D. Walker, and S. M. Clark, Earth Planet. Sci. Lett. 284, 157 (2009).

${ }^{24}$ O. T. Lord, I. G. Wood, D. P. Dobson, L. Vočadlo, W. Wang, A. R. Thomson, E. T. H. Wann, G. Morard, M. Mezouar, and M. J. Walter, Earth Planet. Sci. Lett. 408, 226 (2014)

${ }^{25}$ A. J. Campbell, Rev. Sci. Instrum. 79, 015108 (2008).

${ }^{26}$ T. Watanuki, O. Shimomura, T. Yagi, T. Kondo, and M. Isshiki, Rev. Sci. Instrum. 72, 1289 (2001).

${ }^{27}$ M. J. Walter and K. T. Koga, Phys. Earth Planet. Inter. 143-144, 541 (2004).

${ }^{28}$ L. R. Benedetti and P. Loubeyre, High Pressure Res. 24, 423 (2004).

${ }^{29}$ R. Boehler, H. G. Musshoff, R. Ditz, G. Aquilanti, and A. Trapananti, Rev. Sci. Instrum. 80, 045103 (2009).

${ }^{30}$ G. Shen, L. Wang, R. Ferry, H. K. Mao, and R. J. Hemley, J. Phys.: Conf. Ser. 215, 012191 (2010).

${ }^{31}$ L. Dubrovinsky, K. Glazyrin, C. McCammon, O. Narygina, E. Greenberg, S. Übelhack, A. I. Chumakov, S. Pascarelli, V. Prakapenka, J. Bock, and N. Dubrovinskaia, J. Synchrotron Radiat. 16, 737 (2009).

${ }^{32}$ A. Laskin and V. Laskin, J. Phys.: Conf. Ser. 276, 012171 (2011).

${ }^{33}$ V. B. Prakapenka, A. Kubo, A. Kuznetsov, A. Laskin, O. Shkurikhin, P. Dera, M. L. Rivers, and S. R. Sutton, High Pressure Res. 28, 225 (2008).

${ }^{34}$ W. J. Smith, Modern Optical Engineering, 4th ed. (Tata McGraw-Hill Education, 2007).

${ }^{35}$ J. E. Greivenkamp, Field Guide to Geometrical Optics (SPIE Press, Bellingham, 2004), Vol. 1.

${ }^{36}$ I. Kupenko, C. Strohm, C. McCammon, V. Cerantola, K. Glazyrin, S. Petitgirard, D. Vasiukov, G. Aprilis, A. I. Chumakov, R. Rüffer, and L. Dubrovinsky, Rev. Sci. Instrum. 86, 114501 (2015).

${ }^{37}$ D. L. Heinz and R. Jeanloz, High-Pressure Research in Mineral Physics: A Volume in Honor of Syun-iti Akimoto, Geophys. Monogr. Ser., edited by M. H. Manghnani and Y. Syono (AGU, Washington, D.C., 1987), Vol. 39, pp. 113-127.

${ }^{38}$ S. Petitgirard, A. Salamat, P. Beck, G. Weck, and P. Bouvier, J. Synchrotron Radiat. 21, 89 (2014).

${ }^{39}$ V. Potapkin, A. I. Chumakov, G. V. Smirnov, J. P. Celse, R. Rüffer, C. McCammon, and L. Dubrovinsky, J. Synchrotron Radiat. 19, 559 (2012).

${ }^{40}$ A. I. Chumakov and R. Rüffer, Hyperfine Interact. 113, 59 (1998).

${ }^{41}$ R. Rüffer and A. I. Chumakov, Hyperfine Interact. 128, 255 (2000).

${ }^{42}$ A. A. Maradudin, P. A. Flinn, and S. Ruby, Phys. Rev. 126, 9 (1962).

${ }^{43}$ C. Prescher, C. McCammon, and L. Dubrovinsky, J. Appl. Crystallogr. 45, 329 (2012). 